\title{
Increased myeloid cell hypoxia-inducible factor-1 delays obliterative airway disease in the mouse
}

\author{
Jussi 0. Ropponen, MD, ${ }^{a, b}$ Mikko A. Keränen, $M D, P h D,{ }^{a}$ \\ Alireza Raissadati, MD, ${ }^{a}$ Antti I. Nykänen, MD, PhD, ${ }^{a, b}$ Rainer Krebs, MSc, PhD, \\ Karl B. Lemström, MD, PhD, ${ }^{a, b}$ and Jussi M. Tikkanen, MD, PhD ${ }^{a, b}$
}

\author{
From the ${ }^{a}$ Transplantation Laboratory, Helsinki University, Helsinki, Finland; and the ${ }^{b}$ Cardiac Surgery, Heart and \\ Lung Center, Central Hospital, Helsinki University, Helsinki, Finland.
}

\author{
KEYWORDS: \\ hypoxia-inducible \\ factor-1; \\ obliterative \\ bronchiolitis; \\ lung transplantation; \\ Von Hippel-Lindau \\ protein; \\ allograft rejection
}

\begin{abstract}
BACKGROUND: Obliterative bronchiolitis after lung transplantation is characterized by chronic airway inflammation leading to the obliteration of small airways. Hypoxia-inducible factor-1 (HIF1 ) is a master regulator of cellular responses to hypoxia and inflammation. The Von Hippel-Lindau protein (pVHL) drives the degradation of oxygen-sensitive subunit HIF-1 $\alpha$ that controls the activity of HIF-1. We investigated the effect of myeloid cell-targeted gene deletion of HIF-1 $\alpha$ or its negative regulator $\mathrm{pVHL}$ on the development of obliterative airway disease (OAD) in the recipients of tracheal allografts, a mouse model for obliterative bronchiolitis after lung transplantation.

METHODS: Tracheal allografts were heterotopically transplanted from BALB/c donor mice to fully major histocompatibility complex-mismatched recipient mice with HIF- $1 \alpha$ or VHL gene deletion in myeloid cells. The recipients were left non-immunosuppressed or received tacrolimus daily. Histologic, immunohistochemical, and real-time reverse transcription polymerase chain reaction analyses were performed at 3,10 , and 30 days.

RESULTS: In the absence of immunosuppression, myeloid cell-specific VHL deficiency of the recipient mice improved epithelial recovery, decreased inflammatory cell infiltration and expression of pro-inflammatory cytokines, increased regulatory forkhead box P3 messenger RNA expression, and reduced OAD development in tracheal allografts. In the presence of tacrolimus immunosuppression, loss of HIF- $1 \alpha$ activity in myeloid cells of the recipient by HIF- $1 \alpha$ gene deletion accelerated OAD development in mouse tracheal allografts.

CONCLUSIONS: Activity of the HIF-pathway affects the development of allograft rejection, and our results suggest that myeloid cell-specific VHL-deficiency that potentially increases HIF-activity decreases allograft inflammation and the subsequent development of OAD in mouse tracheal allografts.

J Heart Lung Transplant 2016;35:671-678

(C) 2016 International Society for Heart and Lung Transplantation. All rights reserved.
\end{abstract}

Lung transplantation is the only effective treatment for many end-stage pulmonary diseases. Chronic lung allograft dysfunction (CLAD) is the leading cause of morbidity, lung allograft loss, and late mortality after lung transplantation. ${ }^{1}$ The main known form of CLAD is the classical obliterative

Reprint requests: Jussi O. Ropponen, MD, Transplantation Laboratory, University of Helsinki, PO Box 21 (Haartmaninkatu 3), FI-00014 University of Helsinki, Finland. Telephone: +35-85059-62097.

E-mail address: jussi.o.ropponen@helsinki.fi bronchiolitis (OB), with irreversible fibroproliferation in respiratory bronchioles. ${ }^{2}$ Currently, no effective treatment for CLAD is available. Bronchial epithelial damage and injury of the subepithelial structures seem to be the key initiating event in the development of OB. ${ }^{3,4}$ Ischemiareperfusion injury is one of the most important causes of early allograft injury and may lead to primary graft dysfunction that in turn contributes to the development of OB. ${ }^{5,6}$ Therefore, interventions targeting early immune activation could well result in reduced long-term complications. 
A certain degree of both warm and cold lung ischemia is unavoidable in current clinical practice. The cellular responses to hypoxia are regulated by transcription factors called hypoxia inducible factors (HIFs). ${ }^{7}$ HIF-1 is a principle regulator of hypoxic adaptation, regulating gene expression involved in glycolysis, erythropoiesis, angiogenesis, proliferation, and stem cell function under hypoxia. ${ }^{7}$ HIF-1 is a heterodimer transcription factor constituting of 1 HIF- $\alpha$ sub-unit (HIF- $1 \alpha,-2 \alpha,-3 \alpha)$ and the HIF- $1 \beta$ subunit. HIF-1 is the best described of the $\alpha$ sub-units. The HIF$1 \alpha$ and HIF- $1 \beta$ sub-units are both produced at a constant rate. HIF- $1 \beta$ is stable, but HIF- $1 \alpha$ is extremely fragile in normal oxygen concentrations and is rapidly degraded via a ubiquitin-mediated pathway. ${ }^{8}$ Von Hippel Lindau protein (pVHL) protein is crucial in this process, and its absence leads to stabilization of HIF- $1 \alpha$ and activation of HIF- 1 even in normoxic conditions. ${ }^{9,10}$

Cramer et $\mathrm{al}^{11}$ showed that HIF-1 $\alpha$ plays an important role in the regulation of myeloid cell aggregation, invasion, and motility. Furthermore, the overexpression of HIF-1 $\alpha$ in macrophages leads to enhanced phagocytosis. ${ }^{12}$ On the one hand, HIFs also support the innate immune functions of dendritic cells, mast cells, and epithelial cells. ${ }^{13}$ On the other hand, HIF-1 acts as an adaptive and survival factor for ischemic tissue. $^{14,15}$ Therefore, the final effect of HIF-1 in any one setting is likely dependent on the microenvironment.

Ischemia-reperfusion injury initiates an innate immune response leading to neutrophil and monocyte infiltration into the lung allograft. These monocytes differentiate into antigen-presenting dendritic cells and recruit and activate $\mathrm{T}$ cells, leading to acute alloimmune activation. ${ }^{16}$ Because HIFs have a major role in inflammatory responses during ischemia, we hypothesized that HIF-1 in the allograft infiltrating neutrophils and macrophages may play a major role in innate immune responses and the ensuing alloimmune activation. To test this, we used the heterotopic mouse tracheal allograft model and fully major histocompatibility complex (MHC)-mismatched recipients with myeloid-targeted gene deletion of HIF-1 $\alpha$ or VHL that leads to constant HIF-1 inactivation or activation, respectively. ${ }^{11,17-20}$

\section{Methods}

Permission for animal experimentation in this study was obtained from the Provincial State Office of Southern Finland. Mice received care in compliance with the Guide for the Care and Use of Laboratory Animals prepared by the National Academy of Sciences and published by the National Academy Press (ISBN 0-309-05377, revised 2011).

\section{Heterotopic mouse tracheal allograft transplantation model}

Allogeneic tracheal transplantations were performed from specific pathogen-free fully MHC-mismatched Balb/C (H-2d; Scanbur, Sollentuna, Sweden) to C57BL/6 (H-2b) recipients with HIF-1 $\alpha$ or VHL gene deletion in myeloid cell lineage. HIF-1 $\alpha$ and VHL gene deletion confined to neutrophils and macrophages was originally achieved by mating the Sv129/C57BL/6/CB.20 mice containing loxP sequences on either side of the target gene with Sv129/ C57BL/6/CB.20 mice carrying the LysMCre recombinase. ${ }^{20}$ The resulting mice were backcrossed to wild-type C57BL/6J mice for 12 generations by Professor Randall Johnson (University of Cambridge, Cambridge, United Kingdom), who provided the mice. ${ }^{11}$ The result is a mouse that is deficient in HIF-1 $\alpha$ or VHL alleles in all myeloid cells (later defined as mHIF- $1 \alpha^{-1-}$ or $\mathrm{mVHL}^{-/}$). This was controlled by genotyping the animals used in experiments. Littermates (LMs) served as negative controls. All mice were male, weighed 25-30 grams, and were 2 to 3 months old.

Tracheal allografts were transplanted heterotopically into an abdominal subcutaneous pouch from $\mathrm{Balb} / \mathrm{c}$ donors to gene-deleted or LM recipients. Isoflurane (Baxter, Deerfield, IL) and buprenorphine (Schering-Plough, Kenilworth, NJ) were used for anesthesia and perioperative analgesia. The allografts were removed 3, 10, and 30 days after the transplantation. The number of animals used was 6 per each group.

\section{Drug regimens}

No immunosuppressive or any other treatment was administered in the first phase. In the absence of immunosuppression, the tracheal allografts develop near total luminal occlusion by 30 days. The degree of airway occlusion may be regulated by tacrolimus immunosuppression in a dose-dependent fashion. ${ }^{21}$ In the second phase, the recipients received tacrolimus $0.75 \mathrm{mg} / \mathrm{kg}$ daily subcutaneously that reduces OAD development, and tracheal allografts exhibit approximately $30 \%$ luminal occlusion at 30 days. The dose was based on our previous dose-response study with this animal model. ${ }^{21}$ Tacrolimus was provided by Astellas.

\section{Histologic evaluation}

The transplanted trachea was excised, embedded in Tissue-Tek (Miles Inc, Elkhart, IN), snap frozen in liquid nitrogen, and stored at $-70^{\circ} \mathrm{C}$ until used. For histologic evaluation, frozen sections were stained with Mayer's hematoxylin and eosin. The histologic changes in the respiratory epithelium were evaluated as percentage circumference not covered by epithelium. Luminal occlusion was evaluated as reduction of luminal area using ImageJ 1.59 software (National Institutes of Health, Bethesda, MD). All analyses were done in blinded review by 2 independent observers (J.R. and M.K.).

\section{Immunohistochemistry}

Cryostat sections were stained using the peroxidase avidin-biotinperoxidase complex method (Vectastain Elite ABC Kit; Vector Laboratories, Burlingame, CA), and the reaction was revealed by 3-amino-9-ethylcarbazole (Vector Laboratories). The following antibodies and dilutions were used: myeloperoxidase (MPO; $20 \mu \mathrm{g} / \mathrm{ml}$; catalog number ab9535, Abcam), CD11b (clone M1/70, $5 \mu \mathrm{g} / \mathrm{ml}$ ), CD4 (clone RM4-5, $5 \mu \mathrm{g} / \mathrm{ml}$ ), CD8 (clone 53-6.7, $5 \mu \mathrm{g} / \mathrm{ml}$ ), and CD11c (clone N418, $10 \mu \mathrm{g} / \mathrm{ml}$ ). The number of inflammatory cells was recorded by counting positive-staining cells per cross-section using a grid and original magnification $\times 40$ and moving the grid across the tracheal cross-section in 2 perpendicular lines. All analyses were performed in blinded review by 2 independent observers (J.R. and M.K.). The negative controls showed no immunoreactivity. 


\section{RNA isolation and quantitative real-time polymerase chain reaction}

Total RNA was isolated from a tissue sample dissected from the trachea using RNeasy kit (Qiagen, Hilden, Germany) and reverse transcribed with the High-RNA-to-cDNA kit (Applied Biosystems, Foster City, CA). Quantitative real-time reverse transcription polymerase chain reaction (qRT-PCR) was performed on a RotorGene-6000 (Corbett Research, Doncaster, VIC, Australia) using 2X DyNAmo Flash SYBR Green Master mix (Finnzymes, Espoo, Finland). Measurement of the PCR product was performed at the end of each extension period. All data were normalized against 18sRNA, which was most stably expressed in the respective experiments.

\section{Statistical analyses}

All data are expressed as mean \pm standard error of the mean. The Mann-Whitney $U$ test was used for 2-group comparisons, and the analysis of variance test with Dunnett correction was used for parametric multiple group comparison. Analyses were done with SPSS 15.0 software (IBM Corp, Armonk, NY). Statistical significance was set at $p<0.05$. The mHIF-1 $\alpha^{-1-}$ and $\mathrm{mVHL}^{-/-}$ groups were not compared with each other.

\section{Results}

The development of OAD was delayed in $\mathrm{mVHL}^{-/-}$ recipients of tracheal allografts. In the first phase of our study, we investigated whether the development of OAD in tracheal allografts is altered in $\mathrm{mHIF}-1 \alpha^{-/-}$or $\mathrm{mVHL}^{-/-}$ recipient mice in the absence of immunosuppression. At Day 3, during the ischemic phase, all tracheal allografts showed equally prominent respiratory epithelial cell injury (Figure 1A). The epithelium in $\mathrm{mVHL}^{-/-}$recipients was fully recovered at 10 days and remained partly preserved at 30 days (Figure 1A, C), whereas total loss of epithelium was

\section{B}

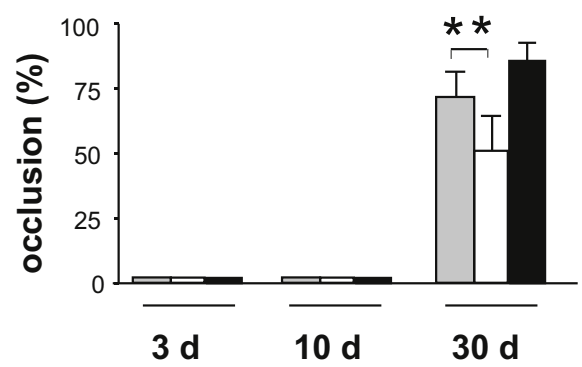

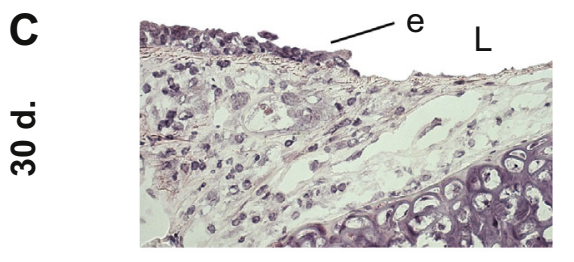

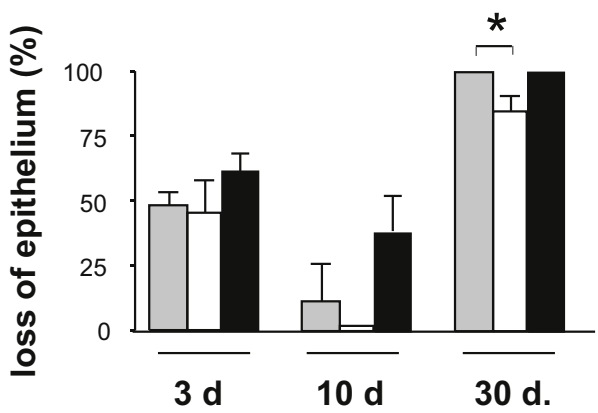

D

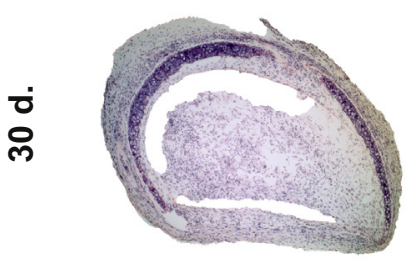

Control
E

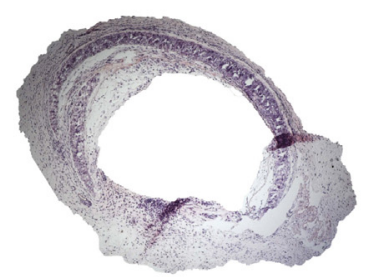

VHL-ko
F

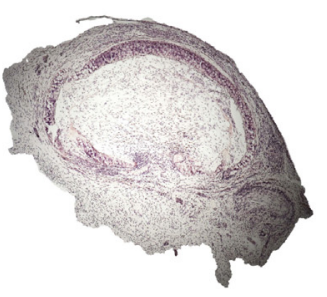

HIF-ko

Figure 1 Development of obliterative airway disease was delayed in Von Hippel-Lindau knockout (VHL ko) mice recipients of tracheal allografts. Tracheal allografts were heterotopically transplanted from Balb/C donors to fully major histocompatibility complex-mismatched recipient mice with hypoxia-inducible factor- $1 \alpha\left(\mathrm{mHIF}-1 \alpha^{-/-}\right)$or VHL $\left(\mathrm{mVHL}^{-/}\right)$gene deletion in myeloid cells. Allografts were analyzed at 3,10 , and 30 days. No immunosuppression was given. (A) The histologic changes in respiratory epithelium were evaluated as the percentage circumference not covered by respiratory epithelium. (C) Illustrative photomicrograph (original magnification $\times 200$ ) stained with Mayer's hematoxylin and eosin. (B) Airway occlusion was evaluated as the reduction in luminal area. (D-F) Photomicrographs (original magnification $\times 80$ ) stained with Mayer's hematoxylin and eosin. Data are expressed as mean \pm standard error of the mean. Statistical analyses was with the analysis of variance test and Dunnett's correction ( $n=6$ per group). mHIF- $1 \alpha^{-/}$and $\mathrm{mVHL}^{-/-}$were not compared with each other. e, epithelium; L, lumen. ${ }^{*} p<0.05$, ${ }^{* *} p<0.005$. 
A
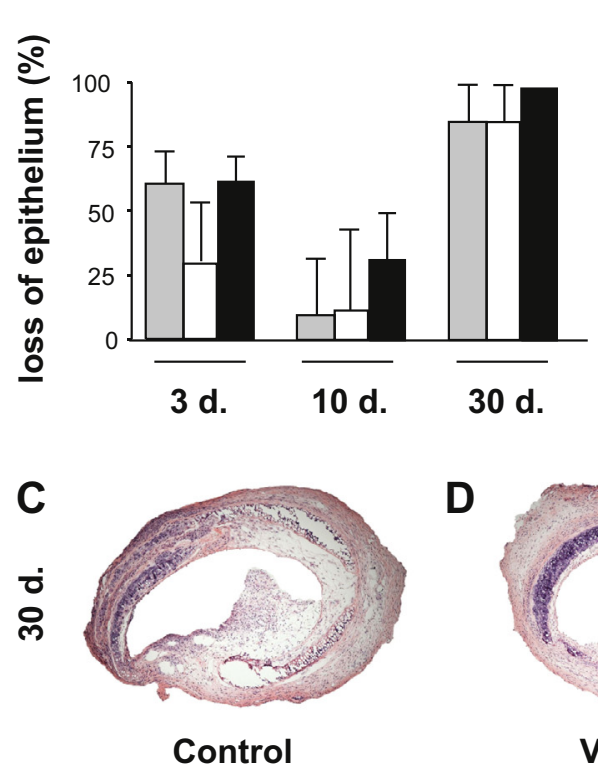

D
B
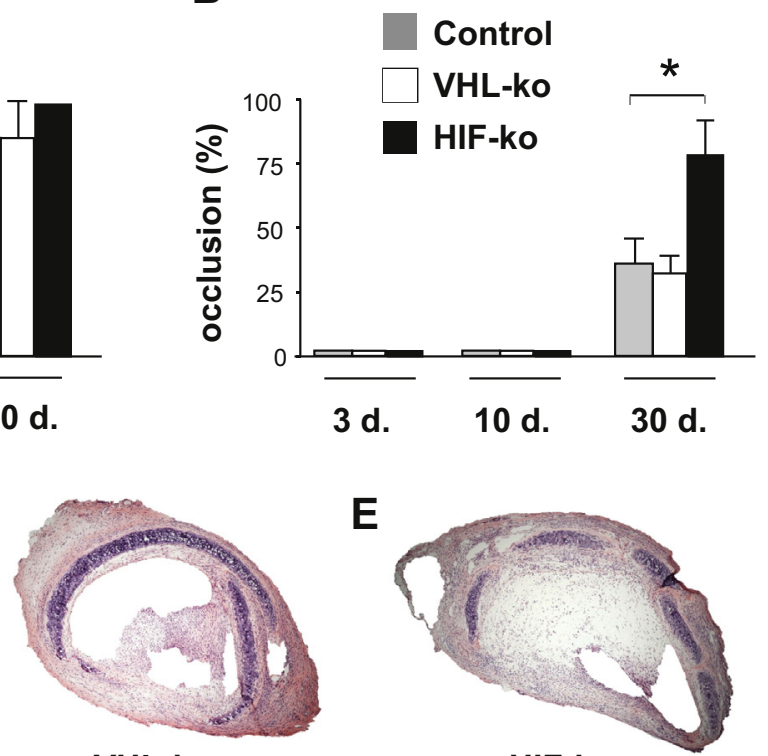

VHL-ko

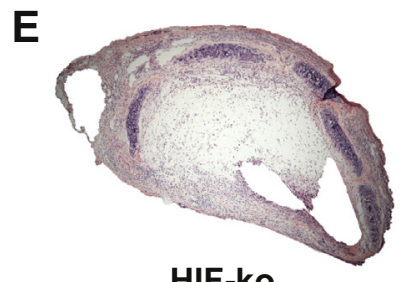

HIF-ko

Figure 2 Hypoxia-inducible factor (HIF) gene deletion in myeloid cells enhances obliterative airway disease in tracheal allografts in the T-cell immunocompromised recipient mice. Tracheal allografts were heterotopically transplanted from Balb/C donors to fully major histocompatibility complex-mismatched recipient mice with hypoxia-inducible factor- $1 \alpha\left(\mathrm{mHIF}-1 \alpha^{-/-}\right)$or Von Hippel-Lindau (mVHL $\mathrm{mL}^{-/-}$) gene deletion in myeloid cells. Allografts were analyzed at 3, 10, and 30 days. The recipient mice received tacrolimus $(0.75 \mathrm{mg} / \mathrm{kg}$ subcutaneously daily). The histologic changes in respiratory epithelium were evaluated as percentage circumference not covered by respiratory epithelium. (B) Luminal occlusion was evaluated as the reduction in luminal area. (C-E) Photomicrographs (original magnification $\times 80)$ stained with Mayer's hematoxylin and eosin. Data are expressed as mean \pm standard error of the mean $(n=6$ per group). mHIF- $1 \alpha^{-/-}$and $\mathrm{mVHL}^{-1-}$ were not compared with each other. Statistical analyses were done with analysis of variance test and Dunnett's correction. ko, knockout. $* p<0.05$.

seen in mHIF- $1 \alpha^{-/-}$recipients and in LMs at 30 days ( $p=$ 0.016 , Figure 1A). No tracheal occlusion was observed in any of the groups at 3 or 10 days (Figure $1 \mathrm{~B}$ ). In $\mathrm{mVHL}^{-/-}$ recipients, the airway occlusion was significantly decreased at 30 days compared with tracheal allografts in LMs $(p=$ 0.001; Figure 1B, D-F).

The development of OAD in tracheal allografts was increased in mHIF- $1 \alpha^{-/-}$recipients in the presence of T-cell immunosuppression. In the second phase of our study, we investigated how changes in HIF-1 activity affect the development of OAD in tracheal allografts in the presence of low-dose tacrolimus. In all allografts, almost total loss of epithelium was seen at 30 days despite immunosuppression (Figure 2A). However, OAD in tracheal allografts was significantly increased in $\mathrm{mHIF}-1 \alpha^{-1-}$ recipients at 30 days $(p=0.02$, Figure $2 \mathrm{~B}-\mathrm{E})$. There was no difference in the degree of preserved epithelium or occlusion between $\mathrm{mVHL}^{-/-}$and LMs (Figure 2A-D).

The infiltration of inflammatory cells in tracheal allografts was reduced in $\mathrm{mVHL}^{-/-}$recipients. The number of $\mathrm{CD}_{11 \mathrm{~b}^{+}}$macrophages and $\mathrm{CD} 4^{+} \mathrm{T}$ cells was significantly reduced in tracheal allografts transplanted to $\mathrm{mVHL}^{-1-}$ recipients at 10 days, $(p=0.024$ and $p=0.014$; Figure 3B, $\mathrm{C}$, and F) compared with LMs. No difference was observed in the number of allograft-infiltrating $\mathrm{MPO}^{+}$neutrophils, $\mathrm{CD} 1 \mathrm{~b}^{+}$macrophages, $\mathrm{CD} 4^{+} \mathrm{T}$ cells, $\mathrm{CD}^{+} \mathrm{T}$ cells, or $\mathrm{CD} 11 \mathrm{c}^{+}$dendritic cells between the groups at 3 days (Figure 3A-E). In the recipient mice treated with tacrolimus immunosuppression, no difference was observed in the number of allograft-infiltrating inflammatory cells between the groups at 3 or 10 days (data not shown).

On the basis of the histologic and immunohistochemical results, we decided to concentrate on $\mathrm{mVHL}^{-1-}$ recipients to define the beneficial effect on the mechanisms of the delayed OAD development.

The messenger $(\mathrm{m})$ RNA expression of proinflammatory cytokines was reduced and T-regulatory cell (Treg) transcription factor was increased in tracheal allografts in $\mathrm{mVHL}^{-1-}$ recipients. On the basis of our previous studies with the tracheal allograft model, ${ }^{22}$ we chose to investigate the mRNA expression of allografts at 10 days, at the time of peak of alloimmune activation. The mRNA expression of Treg transcription factor forkhead box P3 (FoxP3) was significantly increased $(p=0.002)$, meanwhile the mRNA expression of Th17-related cytokine interleukin (IL)-6, monocyte chemotactic protein (MCP)-1, and heme oxygenase-1 (HO-1) was significantly reduced in $\mathrm{mVHL}^{--}(p=$ $0.005, p=0.005$, and $p=0.014$; Figure $4 \mathrm{~A}-\mathrm{C}$ ). There was no significant difference between the groups in the mRNA expression of inflammatory cytokine IL- $1 \beta$ and Th1-related transcription factor T-Bet, cytokines IL-2, IL-12p35, Th2related IL-4, Th17-related transcription factor retinoic acid receptor-related orphan receptor c, Treg-related cytokine IL-10, endothelin- 1 , and growth factors transforming growth factor- $\beta$ or connective tissue growth factor (Figure $4 \mathrm{~A}-\mathrm{C}$ ).

The mRNA expression of Treg transcription factor was increased in the spleens of $\mathrm{mVHL}^{-/}$recipients of tracheal allografts. We also investigated the mRNA expression in the 
A

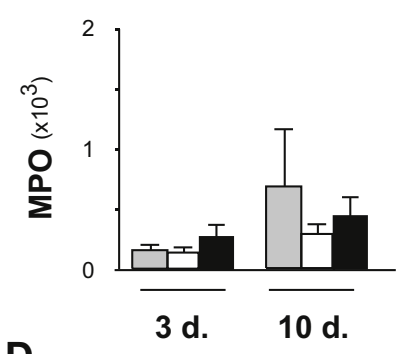

D

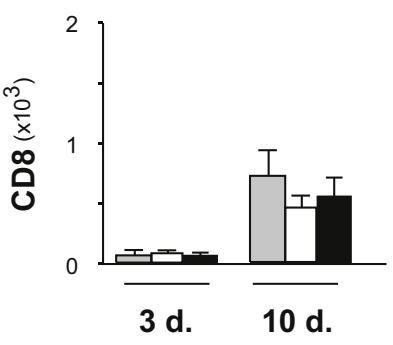

B
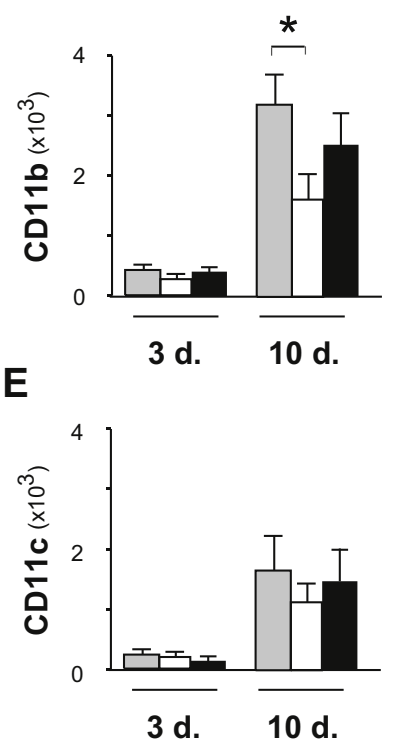

C

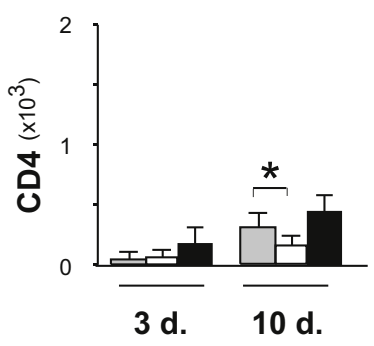

$\mathbf{F}$

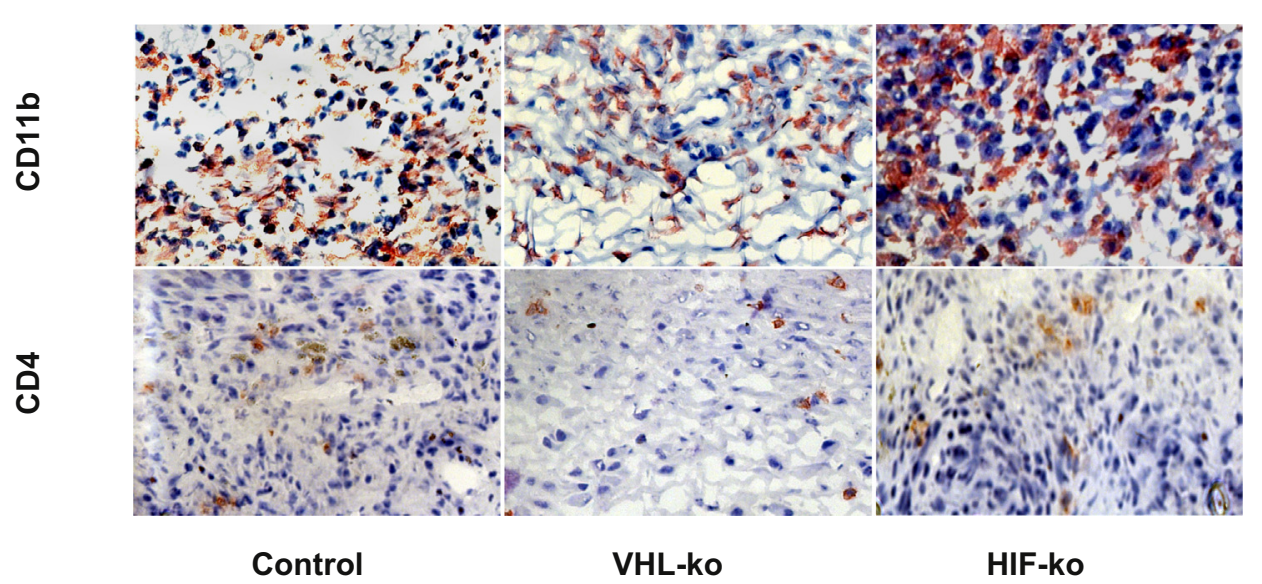

Figure 3 The infiltration of inflammatory cells was reduced in Von Hippel-Lindau knockout (VHL-ko) recipients of tracheal allografts. Tracheal allografts were heterotopically transplanted from Balb/C donors to major histocompatibility complex-mismatched recipients with hypoxia-inducible factor- $1 \alpha\left(\mathrm{mHIF}-1 \alpha^{--}\right)$or VHL $\left(\mathrm{mVHL}^{-/}\right)$deficiency in myeloid cells. Here we show the number $\left(\times 10^{3}\right)$ of (A) myeloperoxidase (MPO) ${ }^{+}$neutrophils, (B) $\mathrm{CD}_{11 \mathrm{~b}^{+}}$macrophages, (C) CD4 ${ }^{+} \mathrm{T}$ cells, (D) $\mathrm{CD} 8^{+} \mathrm{T}$ cells, and (E) CD11 ${ }^{+}$dendritic cells in tracheal cross-sections of allografts at 3 and 10 days. (F) Illustrative photomicrographs (original magnification $\times 200$ ). Data are expressed as mean \pm standard error of the mean. Statistical analyses with analysis of variance test and Dunnett's correction ( $n=6$ per group). mHIF$1 \alpha^{-/-}$and $\mathrm{mVHL}^{-/-}$were not compared with each other. ${ }^{*} p<0.05$.

recipients' secondary lymphoid organ spleen at 10 days. The mRNA expression of MCP-1, IL-1 $\beta$, and FoxP3 was significantly increased in the spleens of the $\mathrm{mVHL}^{-/-}$mice $(p=0.035, p=0.022$, and $p=0.035$; Figure 4D). There was no significant difference in the mRNA expression of HO-1 or IL-6 (Figure 4D).

\section{Discussion}

In this study, we show that myeloid cell-specific deletion of HIF-1 regulating VHL of the recipient mice led to a significant reduction in the OAD development in heterotopic tracheal allografts. In contrast, myeloid cell-specific HIF- $1 \alpha$ gene deletion accelerated $\mathrm{OAD}$ development even in the presence of T-cell inhibition with tacrolimus. These results suggest a beneficial role for myeloid cell-specific VHLdeficiency in this model.

In this heterotopic non-vascularized model, tracheal neovascularization is preceded by an ischemic phase for the first 2 to 3 days after transplantation, resulting in ischemic injury to the respiratory epithelium. ${ }^{17,22}$ In syngrafts, ischemia induces a self-limiting, alloantigenindependent innate immune response, the epithelium recovers completely, and no OAD develops. ${ }^{22}$ In the allografts without immunosuppression, the early ischemic injury is followed by a predominantly pro-inflammatory milieu. Alloantigen-dependent Th1 and Th17 responses peak at 7 to 10 days, followed by progressive epithelial damage and a nearly total airway occlusion 30 days after transplantation. ${ }^{22}$ 
A

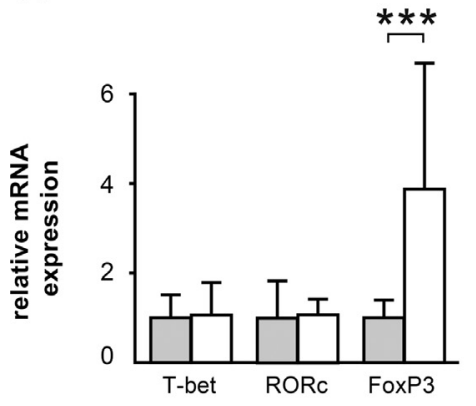

C

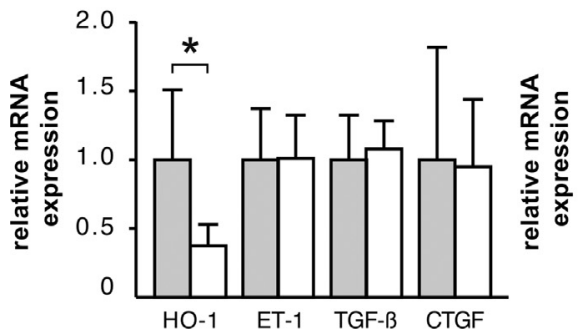

B

D
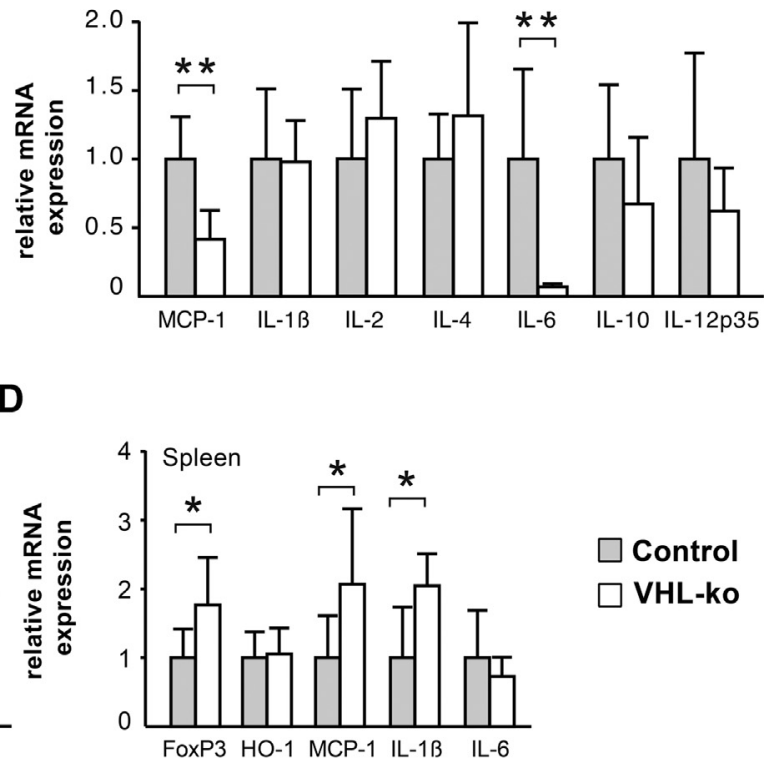

Figure 4 The messenger RNA (mRNA) expression of T-regulatory cell transcription factor was increased in tracheal allografts and spleens of Von Hippel-Lindau knockout (VHL-ko) recipients. Tracheal allografts were heterotopically transplanted from Balb/C donors to major histocompatibility complex-mismatched recipients with VHL $\left(\mathrm{mVHL}^{-1-}\right)$ deficiency in myeloid cells. Quantitative reversetranscription polymerase chain reaction analysis was performed from the (A-C) tracheal samples and from the (D) spleens 10 days after transplantation. mRNA data were normalized against 18sRNA and represented as a fold-increase compared with tracheal allografts in littermate control mice. Data are expressed as mean \pm standard error of the mean ( $n=6$ per group). Statistical analyses with Mann-Whitney $U$ test. CTGF, connective tissue growth factor; ET, endothelin; FoxP3, forkhead box P3; HO, heme oxygenase; IL, interleukin; MCP, monocyte chemotactic protein; RORc, retinoic acid receptor-related orphan receptor; TGF, transforming growth factor. $* p<0.05, * * p<$ $0.01, * * * p<0.005$.

Inflammatory cells play a major role in the development of immune responses, and because of this we chose to use mice with HIF-1 $\alpha$ and VHL gene deletion in myeloid cells only in this study. The development of OAD was seen all groups, which was not a surprise considering the robust nature of the model where the trachea is transplanted over a major MHC mismatch. Without any immunosuppression we were able to investigate the beneficial effects of VHL deficiency. HIF-1 $\alpha$ expression was previously reported to have an inhibitory role in T-cell functioning. ${ }^{23}$ In our study, myeloid cell-specific VHL deficiency in the recipients reduced accumulation of $\mathrm{CD} 11 \mathrm{~b}^{+}$macrophages and $\mathrm{CD} 4^{+}$ $\mathrm{T}$ cells in mouse tracheal allografts during the peak of the alloimmune response at 10 days. In addition, the mRNA expression of pro-inflammatory cytokines MCP-1, IL-6, and $\mathrm{HO}-1$ was also significantly reduced in $\mathrm{mVHL}^{-/-}$recipients. However, even low-dose tacrolimus preventing T-cell signal transduction and IL-2 production ${ }^{21}$ did not delay the development of OAD in the mHIF- $1 \alpha^{-/-}$recipients.

There are a few plausible mechanisms of action by which myeloid cell-specific VHL deficiency reduced OAD in our study. VHL deficiency likely leads to increased HIF-1 activity and thus was associated with a lesser degree of epithelial loss at 10 days. Because the rate of epithelial loss was similar in all groups at 3 days, it is unlikely that early ischemia-related epithelial injury was affected by myeloid cell HIF-1 expression. In this model, even the epithelium in syngrafts suffers extensive early damage and then recovers in the absence of alloimmune activation. ${ }^{22}$ Earlier, the increased vascular endothelial growth factor-A expression was shown to improve epithelial recovery in this model. ${ }^{24}$ Furthermore, allograft overexpression of HIF- $1 \alpha$ promotes repair of airway microvasculature through the induced expression of pro-angiogenic factors in mice. ${ }^{25}$ However, we could not demonstrate pro-angiogenic effects in $\mathrm{mVHL}^{-1-}$ mice in our study (data not shown). It is more likely that the markedly reduced acute inflammatory response allowed epithelial regeneration in tracheal allografts. As a result, the complete loss of the tracheal epithelium was delayed in $\mathrm{mVHL}^{-/-}$mice. The importance of epithelial injury in OAD development is well established, ${ }^{3,26}$ and reduced injury may explain the delay in OAD development in our study. However, epithelial regeneration does not explain the reason for initially reduced inflammatory response.

The cytokine IL-6 is a crucial mediator in priming naïve $\mathrm{T}$ cells toward the Th17 phenotype that is important in adaptive immune responses on epithelial surfaces. ${ }^{27}$ Recent studies show that IL-6 is also a potent inhibitor of transforming growth factor- $\beta$-driven induction of $\mathrm{CD}^{+}$ FoxP3 $^{+}$Tregs. ${ }^{28}$ Interestingly, we observed reduced mRNA expression of IL-6 and simultaneously increased mRNA expression of FoxP3 in $\mathrm{mVHL}^{-1-}$ allografts in this study. Furthermore, we also observed reduced mRNA expression of MCP-1 and reduced allograft infiltration of inflammatory cells into the allograft. Altogether, these results suggest a decreased adaptive immune response in $\mathrm{mVHL}^{-/-}$and Treg 
pathway activation in these allografts at 10 days. Although concomitantly increased mRNA expression of proinflammatory cytokines was seen in the spleens of $\mathrm{mVHL}^{-/-}$, increased FoxP3 expression was also observed, suggesting increased Treg activity also in the secondary lymphoid organ. Ben-Shoshan et $\mathrm{al}^{29}$ recently reported that hypoxia controls Treg homeostasis via HIF-1. The number as well as the potency of Tregs are also enhanced in hypoxia. ${ }^{29}$ In our study, VHL-deficiency in the myeloid cells may have resulted in increased Treg activity leading to reduced inflammatory and immune responses. However, we also observed decreased HO-1 expression in $\mathrm{mVHL}^{-/}$ during the peak alloimmune response at 10 days. On the one hand, this is somewhat at odds with reports suggesting HO1 is protective in the setting of transplant rejection where HO-1 has been reported to have anti-apoptotic and protective effects. ${ }^{30}$ On the other hand, increased HO-1 expression is also linked to the development of chronic rejection in heart transplantation. ${ }^{31}$ In our study, reduced HO- 1 expression at the time of peak inflammation and acute rejection is likely to be secondary to the reduction of the number of T cells as HO-1 expression is increased during acute T-cell-mediated rejection in lung allografts. ${ }^{32,33}$

We acknowledge that $\mathrm{mVHL}^{-/-}$leads to stabilization of both HIF isoforms, HIF- $1 \alpha$ and HIF- $2 \alpha .{ }^{10}$ However, we did not dissect which of the isoforms is responsible for the beneficial effect, and furthermore, we did not compare $\mathrm{mVHL}^{-/-}$directly to HIF- $1 \alpha^{-/-}$but separately to the control. Previous studies showed that macrophages and neutrophils are able to express both HIF- $1 \alpha$ and HIF- $2 \alpha{ }^{34,35}$ However, because the OAD development was significantly accelerated in $\mathrm{mHIF}-1 \alpha^{-/-}$recipients, one may assume that HIF-1 activity was the major contributor to the decreased OAD development in $\mathrm{mVHL}^{-1-}$ recipients. In addition, Thompson et $\mathrm{al}^{35}$ reported that HIF- $2 \alpha$ regulates mainly key neutrophil functions, but we did not detect any significant difference in the number of $\mathrm{MPO}^{+}$neutrophils between the groups at 3 or 10 days in our study.

The tracheal allograft model used in our study has several limitations that should be kept in mind when interpreting the results. Firstly, the trachea differs anatomically from the bronchiole and is not in contact with the environment. ${ }^{17}$ Also, this is a non-vascularized model, and therefore, the early ischemic injury is very severe. ${ }^{36}$ Owing to the robust nature of this model, the loss of epithelium and OAD development are much more accelerated compared with the clinical setting. In addition, the acute and rapid development of the obliterative lesion compared with the slower and more chronic process seen in clinical BOS may limit extrapolation of treatment effects into the clinic. However, despite the model's shortcomings, it has the advantage of being easily reproduced, and the obliterative lesion resembles that of the fibroproliferative lesions seen in human OB. ${ }^{17}$ In addition, we used knockout animals with HIF-1 $\alpha$ or VHL deficiency in myeloid cells. However, one should keep in mind, that although most of the myeloid cells have a targeted deletion, the deletion efficiency of HIF-1 $\alpha$ or VHL is not $100 \% .^{11}$

In conclusion, VHL-deficiency in myeloid cells improves epithelial recovery, reduces allograft inflammation, and increases regulatory FoxP3 mRNA expression in mouse tracheal allografts. Importantly, this results in betterpreserved tracheal epithelium and a decrease in OAD development. In contrast, knocking out myeloid cell expression of HIF-1 accelerates OAD development in mice receiving low-dose tacrolimus therapy. Our results suggest that manipulation of the HIF-1 pathway may have a protective role in preventing $\mathrm{OAD}$ in an experimental model for lung transplantation.

\section{Disclosure statement}

None of the authors has a financial relationship with a commercial entity that has an interest in the subject of the presented manuscript or other conflicts of interest to disclose.

The authors thank Eeva Rouvinen, RN, for her excellent technical assistance.

This study was supported by grants from Helsinki University Central Hospital Research Funds, the Sigrid Juselius Foundation, the Academy of Finland, the Research and Science Foundation of Farmos, The Paulo Foundation, Jalmari and Rauha Ahokas Foundation, Aarne Koskelo Foundation, Päivikki and Sakari Sohlberg Foundation, Jane and Aatos Erkko Foundation, Orion Research Foundation, and the University of Helsinki.

\section{References}

1. Verleden GM. Chronic allograft rejection (obliterative bronchiolitis). Semin Respir Crit Care Med 2001;22:551-8.

2. Woodrow JP, Shlobin OA, Barnett SD, Burton N, Nathan SD. Comparison of bronchiolitis obliterans syndrome to other forms of chronic lung allograft dysfunction after lung transplantation. J Heart Lung Transplant 2010;29:1159-64.

3. Belperio JA, Weigt SS, Fishbein MC, Lynch JP 3rd. Chronic lung allograft rejection: mechanisms and therapy. Proc Am Thorac Soc 2009;6:108-21.

4. Glanville AR, Aboyoun CL, Havryk A, Plit M, Rainer S, Malouf MA. Severity of lymphocytic bronchiolitis predicts long-term outcome after lung transplantation. Am J Respir Crit Care Med 2008;177:1033-40.

5. King RC, Binns OA, Rodriguez F, et al. Reperfusion injury significantly impacts clinical outcome after pulmonary transplantation. Ann Thorac Surg 2000;69:1681-5.

6. Fiser SM, Tribble CG, Long SM, et al. Ischemia-reperfusion injury after lung transplantation increases risk of late bronchiolitis obliterans syndrome. Ann Thorac Surg 2002;73:1041-7: discussion 1047-8.

7. Imtiyaz HZ, Simon MC. Hypoxia-inducible factors as essential regulators of inflammation. Curr Top Microbiol Immunol 2010;345: 105-20.

8. Salceda S, Caro J. Hypoxia-inducible factor 1alpha (HIF-1alpha) protein is rapidly degraded by the ubiquitin-proteasome system under normoxic conditions. Its stabilization by hypoxia depends on redoxinduced changes. J Biol Chem 1997;272:22642-7.

9. Jaakkola P, Mole DR, Tian YM, et al. Targeting of HIF-alpha to the Von Hippel-Lindau ubiquitylation complex by O2-regulated prolyl hydroxylation. Science 2001;292:468-72.

10. Krieg M, Haas R, Brauch H, Acker T, Flamme I, Plate KH. Upregulation of hypoxia-inducible factors HIF-1alpha and HIF-2alpha under normoxic conditions in renal carcinoma cells by Von HippelLindau tumor suppressor gene loss of function. Oncogene 2000;19: 5435-43.

11. Cramer T, Yamanishi Y, Clausen BE, et al. HIF-1alpha is essential for myeloid cell-mediated inflammation. Cell 2003;112:645-57.

12. Anand RJ, Gribar SC, Li J, et al. Hypoxia causes an increase in phagocytosis by macrophages in a HIF-1alpha-dependent manner. J Leukoc Biol 2007;82:1257-65. 
13. Nizet V, Johnson RS. Interdependence of hypoxic and innate immune responses. Nat Rev Immunol 2009;9:609-17.

14. Semenza GL. Surviving ischemia: adaptive responses mediated by hypoxia-inducible factor 1. J Clin Invest 2000;106:809-12.

15. Prabhakar NR, Semenza GL. Adaptive and maladaptive cardiorespiratory responses to continuous and intermittent hypoxia mediated by hypoxia-inducible factors 1 and 2. Physiol Rev 2012;92: 967-1003.

16. Goldstein DR, Palmer SM. Role of Toll-like receptor-driven innate immunity in thoracic organ transplantation. J Heart Lung Transplant 2005;24:1721-9.

17. Sato M, Keshavjee S, Liu M. Translational research: animal models of obliterative bronchiolitis after lung transplantation. Am J Transplant 2009:9:1981-7.

18. Hele DJ, Yacoub MH, Belvisi MG. The heterotopic tracheal allograft as an animal model of obliterative bronchiolitis. Respir Res 2001;2: 169-83.

19. McDyer JF. Human and murine obliterative bronchiolitis in transplant. Proc Am Thorac Soc 2007;4:37-43.

20. Clausen BE, Burkhardt C, Reith W, Renkawitz R, Forster I. Conditional gene targeting in macrophages and granulocytes using LysMcre mice. Transgenic Res 1999;8:265-77.

21. Hollmen M, Tikkanen JM, Nykanen AI, Koskinen PK, Lemstrom KB. Tacrolimus treatment effectively inhibits progression of obliterative airway disease even at later stages of disease development. J Heart Lung Transplant 2008;27:856-64.

22. Ropponen JO, Syrjala SO, Krebs R, Nykanen A, Tikkanen JM, Lemstrom KB. Innate and adaptive immune responses in obliterative airway disease in rat tracheal allografts. J Heart Lung Transplant 2011;30:707-16

23. Lukashev D, Ohta A, Sitkovsky M. Hypoxia-dependent anti-inflammatory pathways in protection of cancerous tissues. Cancer Metastasis Rev 2007;26:273-9.

24. Krebs R, Tikkanen JM, Nykanen AI, et al. Dual role of vascular endothelial growth factor in experimental obliterative bronchiolitis. Am J Respir Crit Care Med 2005;171:1421-9.
25. Jiang X, Khan MA, Tian W, et al. Adenovirus-mediated HIF-1alpha gene transfer promotes repair of mouse airway allograft microvasculature and attenuates chronic rejection. J Clin Invest 2011;121: 2336-49.

26. Neuringer IP, Aris RM, Burns KA, Bartolotta TL, Chalermskulrat W, Randell SH. Epithelial kinetics in mouse heterotopic tracheal allografts. Am J Transplant 2002;2:410-9.

27. Miossec P, Korn T, Kuchroo VK. Interleukin-17 and type 17 helper t cells. N Engl J Med 2009;361:888-98.

28. Bettelli E, Carrier Y, Gao W, et al. Reciprocal developmental pathways for the generation of pathogenic effector Th17 and regulatory T cells. Nature 2006;441:235-8.

29. Ben-Shoshan J, Maysel-Auslender S, Mor A, Keren G, George J. Hypoxia controls cd $4+\mathrm{cd} 25+$ regulatory t-cell homeostasis via hypoxia-inducible factor-1alpha. Eur J Immunol 2008;38:2412-8.

30. Soleymaninejadian E, Pramanik K, Samadian E. Immunomodulatory properties of mesenchymal stem cells: cytokines and factors. Am J Reprod Immunol 2012;67:1-8.

31. Holweg CT, Balk AH, Snaathorst J, et al. Intragraft heme oxygenase-1 and coronary artery disease after heart transplantation. Transpl Immunol 2004; 13:265-72.

32. Song R, Kubo M, Morse D, et al. Carbon monoxide induces cytoprotection in rat orthotopic lung transplantation via anti-inflammatory and antiapoptotic effects. American J Pathol 2003;163:231-42.

33. Lu F, Zander DS, Visner GA. Increased expression of heme oxygenase-1 in human lung transplantation. J Heart Lung Transplant 2002;21:1120-6.

34. Poitz DM, Augstein A, Hesse K, et al. Regulation of the HIF-system in human macrophages - differential regulation of HIF-alpha subunits under sustained hypoxia. Mol Immunol 2014;57:226-35.

35. Thompson AA, Elks PM, Marriott HM, et al. Hypoxia-inducible factor 2alpha regulates key neutrophil functions in humans, mice, and zebrafish. Blood 2014;123:366-76.

36. Koskinen PK, Kallio EA, Krebs R, Lemstrom KB. A dose-dependent inhibitory effect of cyclosporine A on obliterative bronchiolitis of rat tracheal allografts. Am J Respir Crit Care Med 1997;155:303-12. 